\title{
Potential Effects of New Zealand's Policy on Next Generation High-Speed Access Networks
}

\author{
Kay E. Winkler * \\ ISCR Working Paper \\ May 2014
}

\begin{abstract}
New Zealand's strategy to deploy ultra-fast next generation access networks (NGA) on the basis of fibre to the home (FTTH) to the majority of the population by 2019 involves specific public private partnerships for dedicated roll-out areas that are supported with substantial financial aid by the Crown. This article explores in which way this strategy can be effective and whether it is able to accelerate consumer demand for NGA. Several empirical studies relating to the deployment and uptake of broadband technology consistently reveal factors which are decisive for the diffusion of broadband technologies in developed countries. From the supply-side perspective, the regulatory environment, associated incentives to deploy new infrastructure, and government stimuli can be seen as important determinants. However, the diffusion of a new technology in a given market requires consumer acceptance. The consumer uptake of ultra-fast broadband (UFB) access will depend on the increase in speed in relation to the existing access technology, and the existence of applications requiring this increase. Taking these factors into account, some potential problems with New Zealand's rollout plan can be identified. It seems conceivable that the driving factor for fast broadband uptake in New Zealand is, under the current set of applications, the migration from low bandwidth broadband to higher bandwidths required for video streaming, but not necessarily to ultra-fast broadband. In that sense, a diminishing marginal return of speed may be assumed. Further, the regulatory environment might cause adverse effects for competing broadband networks that are not subsidized, such as the recently rolled out VDSL network and $4 G$ mobile networks. Moreover, the incentives of retail service providers to offer fibre based internet products are not clear cut. Because of vertical separation they are not invested in network deployment. An empirical analysis of recent UFB uptake data could show whether these assumptions are valid.
\end{abstract}

\footnotetext{
* Kay E. Winkler is a Research Assistant at the New Zealand Institute for the Study of Competition and Regulation (ISCR).
} 
Fibre-optical next generation access (NGA) networks lately have attracted a great deal of attention of policy makers around the world. It is generally believed that bringing fibre networks closer to premises will provide impetus for the economy, and be essential for modern societies in the coming decades. Hence, the wide-spread deployment of NGA has become a policy priority in many countries, and New Zealand is one of them. In 2009, its government started an investment programme that involves subsidies and public private partnerships to foster development of NGA and has the objective to make fibre to the home (FTTH) networks available to $70 \%$ of New Zealanders by 2019 . Being roughly half-way through that programme, it is time to reflect on the effects of the broadband policies on fibre access network deployment, and also to reconsider the expectations about the demand of the new technology by consumers.

Several factors are relevant for the success of telecommunications networks, and more specifically of NGA. From the supply-side perspective, the regulatory environment, associated incentives to deploy new infrastructure, and government stimuli can be seen as important determinants. However, the diffusion of a new technology in a given market depends on consumer acceptance, or uptake by consumers, measured in penetration levels. Simply because consumers have the option to buy NGA, does not mean that they are inclined to do so. New Zealand's NGA initiative addresses consumer demand only to a limited extent, and has as its main goal the mere deployment of NGA, not its acceptance by consumers. However, from an economic perspective, it must ultimately be evaluated on the increase of welfare it generates, and that will depend on the degree to which it accelerates NGA diffusion.

This paper sketches out the framework that governs the diffusion of NGA networks. By reviewing a large body of existing empirical research, it can identify several factors that seem generally applicable to the diffusion of broadband networks. Recent literature offers valuable insight into the question of which factors determine broadband uptake and can be expected to be influential for the diffusion of New Zealand's NGA as well. The first and main part of this paper summarizes the empirical literature on the subject of broadband diffusion and highlights the essential elements for an economic framework of NGA uptake. The second part discusses briefly in which way that framework is applicable to the situation in New Zealand. Some factors will be identified that seem to be relevant, but further research is needed to make more accurate predictions of the potential effects of New Zealand's ultra-fast broadband policy. 
There is plenty of literature dealing with determinants that influence the penetration of new telecommunications services in society. The following sections concentrate on empirical work, demonstrating that several elements of technology diffusion are in fact identifiable in telecommunications markets world-wide. The first set of literature defines the patterns of diffusion that can be observed for technological innovation in general and also in the telecommunications sector. The second body of research assesses several determinants for the speed of diffusion on the example of broadband technology. More recent literature is able to retrospectively analyse empirical evidence, which is important to predict influencing factors for fibre diffusion. The last section will summarize the findings of recently published articles in the past three to four years. This research evaluates specifically the impact of access regulation on both investments in fibre access networks and adoption of related high-speed broadband services.

\subsection{Theoretical Framework on Diffusion of Innovation in Telecommunications}

In general terms, diffusion models are used to forecast the adoption of new technologies by consumers, or in other words the penetration of innovations in society. Those models are useful for the management of firms when deciding on investment strategies in a given region, as well as for governments and regulators in finding optimal policies for shaping markets in innovative industries.

Diffusion processes which were captured in mathematical models to allow the forecasting of demand are likely to have sigmoid shapes (Bass, 1969; Rogers, 2003). These algorithms depict the product life-cycle in a symmetric curve in which half of the market saturation is completed in half of the time. It has been shown, however, that these functions overestimate the adoption rate prior to the $50 \%$ saturation point (Meade and Islam, 2001). To account for asymmetric diffusion curves, the initial models have been adapted in various ways, leading to highly complex models. The difficulty with more complex models is that their forecast accuracy is lower than less complex models (Meade and Islam, 2001). This is problematic where data are limited, which is usually the case with innovative products when adoption just begins. As an alternative, the Gompertz model can be used as a forecasting tool that is less complex and more easily applied when a limited amount of data is available (Gregg, Hassel and Richardson, 1964).

In telecommunications markets, diffusion models are first and foremost used as marketing tools to predict the demand for new products and their life-cycle (Meade and 
Islam, 2006; Fildes and Kumar, 2002). However, these models can also provide useful information for policy makers regarding the choice of appropriate regulatory decisions (Karaçuka and Çatik, 2012).

It is generally accepted that S-shaped, or sigmoid, models are appropriate as a first step towards analysing the diffusion of telecommunications services (Gruber and Verboven, 2001; Rouvinen, 2006; Sundqvist et al., 2005; Wu and Chu, 2010). The sigmoid curve indicates different stages of the product life-cycle in telecommunications. During the initial phase the product is introduced and is only adopted slowly by customers. With increasing adoption, the information about the new product spreads and the uptake of customers accelerates accordingly. When the diffusion gets closer to saturation, the curve reaches an inflection point that marks the maturity stage in which the uptake slows down again (Golder and Tellis, 2004). The initially slow and then increasingly accelerated adoption of telecommunications products resembles the spread of epidemics which can usually be depicted with sigmoid curves as well (Meade and Islam, 2006).

There are several growth models having sigmoid diffusion curves that may be applicable to telecommunications products. Meade and Islam (2001) examine 17 growth models based on time series, and establish that those models that use fewer variables are superior to forecast diffusion in the telecommunications sector. In accordance with their findings, most authors find Logistic, Gompertz or Bass models suitable for analysing diffusion of telecommunications products (Dekimpe et al., 1998; Gruber and Verboven, 2001; Lee and Cho, 2007; Rouvinen, 2006; Sundqvist et al., 2005). Wu and Chu (2010) conclude that different models may fit to different stages of life-cycles: before diffusion take-off the Gompertz model is superior, whilst the Logistic model outperforms other models after the inflection point and over the complete life-cycle of telecommunications products.

For telecommunications services in Turkey, Karaçuka and Çatik (2012) assess the ability of product growth models to forecast diffusion. They observe that the variable exponential growth model is better suited for the diffusion of fixed line services. This suggests that S-shaped models cannot predict the period of the maturity stage. On the other hand, they find that the Gompertz model can better explain diffusion relating to mobile telecommunication services. They conclude that the explanatory powers of different growth models depend on the characteristics of the products and their life-cycle spans, as well as on the competitiveness of the market and its structure. Since strong network effects are indicated for telecommunications services, new technologies should be promoted by governments until a critical mass is achieved, and policy measures should be implemented 
to prevent the abuse of market power.

Michalakelis et al. (2010) extend the classical diffusion framework with a "diffusionprice" model to account for the influence of prices on penetration rates and vice-versa. They estimate price and demand elasticities for several European countries based on figures from the years 2003-2005 and predict how these interact. Price indices and hedonic functions are combined with a diffusion function in order to forecast diffusion of technology innovations in telecommunications. They state that price influences demand to a higher extent than the other way around: "It is obvious that by setting a lower price demand increases, but the vice versa can be the case only for the amount that makes the marginal cost of production equal the marginal profit" (p. 235). Their model predicts a declining pricing path, while diffusion increases more rapidly than shown with a classical diffusion model, which is based on the elapsed time as the only diffusion variable. The estimations are confirmed by actual prices.

\subsection{Empirical Studies on Broadband Diffusion}

There is an increasing body of research analysing the state of broadband diffusion in various countries. The studies interpret data related to broadband uptake and compare them to diffusion models, and to some degree also discuss explanations for the diffusion effects analysed.

An early study of broadband diffusion forecasts future demand of high-speed internet access in the US (Vanston, 2002). It uses the Gompertz model and estimates the speed of adoption value by averaging the adoption speed of historical analogies, such as radio, television, pay cable, mobile phones and internet access. From this, the study forecasts that $25 \%$ of households will subscribe to high-speed internet by 2005 , and $50 \%$ by 2010 . Over this period, an evolution of data rates to an average of $10 \mathrm{Mbit} / \mathrm{s}$ is expected, advancing to over $50 \mathrm{Mbit} / \mathrm{s}$ by 2015.

Concentrating on competition as a determinant of broadband diffusion, Denni and Gruber (2007) conclude that the form of competition matters. They use semi-annual data of the US for the period from 1999 to 2005 , and apply the logistic diffusion function. They find that inter-platform competition has a significant and positive impact on diffusion speed. Contrarily, competition on the platform has a negative, or at least ambiguous, effect. The authors suggest that firm size matters, and diffusion rates are higher when not too many entrants challenge the incumbent, and are relatively large sized firms. Also, intra-platform competition could reduce the investment incentives of incumbents. Other studies comparing 
data from European or OECD countries confirm the result that inter-platform competition between Cable and DSL, or even FTTH, is more significant for broadband diffusion than intra-platform competition (Bouckaert et al., 2010; Cava-Ferreruela and Alabau-Munoz, 2006; Distaso et. al, 2005; Grosso, 2006; Tsuji et al. 2012; Wallsten, 2006).

Based on survey data of households in the United Kingdom, Robertson et al. (2007) estimate broadband adoption curves for different income segments. They notice that diffusion models usually assume an equal probability of all consumers to adopt a product with its introduction. In their view, this assumption is unrealistic as early adopters can be different from late adopters, especially in terms of income. Segmentation of different income groups could provide more accurate forecasts than aggregate approaches. Using the Gompertz model, they are able to estimate broadband adoption curves for different household income levels. As expected, they demonstrate that broadband penetration levels are income sensitive. There are consistent patterns showing that highest income households report a much faster adoption than the lowest. This might explain the effect of the digital divide. Further, their model predicts that overall household adoption of broadband will increase when lower income groups migrate to higher income categories.

Income, education, and population density were identified as statistically significant demographic variables by Lee, Marcu and Lee (2011). The study applies the logistic regression model on annual data for 30 OECD countries covering the years 2000 to 2008 . The main goal was to examine wether LLU had a positive effect on broadband diffusion. The authors conclude that the speed of diffusion appears to increase with LLU, but could not rule out that incentives to invest and long-term expected numbers of subscribers are negatively affected. The simultaneous analysis of mobile broadband diffusion reveals that in many OECD countries, mobile services appear to be a complement to fixed broadband. This could be explained with the high income level in OECD countries.

Unlike other studies, Belloc et al. (2012) examine the effect of both supply-side and demand-side policies on broadband diffusion. Supply-side policies include (amongst others) long-term loan and financing programs, and the creation of public-private-partnerships. Demand-side policies relate to public demand, incentives to business, and private demand such as educational programs, demand subsidies for low-income customer groups, as well as aggregation of potential subscribers to facilitate economies of scale. The estimations are based on a four-step analysis involving OLS and quantile regression. Data are mainly derived from a dataset that contains information on broadband policies adopted by 30 OECD countries over the years 1995 to 2010 . The authors observe that demand-side policies have 
in total a greater impact than supply-side intervention. This result might seem surprising, given that most national policies focus on the supply-side. A further outcome of the study is that the importance of the policies changes during the diffusion process. The influence of demand-side policies increases in the later stages of broadband penetration. It seems that demand-side incentives become more effective with greater availability of infrastructure. Also, network effects might explain this result. On the other hand, supply-side policies lose their relevance with increasing broadband diffusion. Consumers with a lower willingness to adopt broadband become harder to reach as the market matures. Similarly, the effect of the degree of competition is non-linear. The factor of competition is more important during the initial and the advanced phase, and plays a less significant role in the intermediate stage of broadband diffusion. The authors explain this result with the fact that competition enhances the aggregate level of investment in the initial stage. During the intermediate stage, competitors lower prices to acquire customers, which also results in customers switching operators, and the effect on acquiring new customers is reduced. In the final stage, the pricing strategies of competing operators might be exhausted so that they are forced to seek new customers by extending the coverage of the networks.

Broadband data on regional level are analysed by Dauvin and Grzybowski (2013). Their study is based on broadband penetration data for 96 so-called NUTS1 regions of 27 EU countries, expressed as subscriptions per households. In order to estimate effects of competition, the analysis makes use of country-level household survey data for approximating inter-platform competition, and country-level prices for LLU as well as the incumbent's share in DSL-connections for measuring intra-platform competition. Applying linear demand specifications and the logistic diffusion function, they find that both intra- and inter-platform competition have significant impact on broadband diffusion. Higher $\mathrm{HHI}$ values for inter-platform competition leads to lower broadband penetration. Similarly, broadband penetration is lower in countries with a higher incumbent DSL share, as well as in countries with higher LLU prices. They also found socioeconomic factors were relevant, such as income, eduction, population density, and computer penetration. The relevance was of varying degrees, depending on the applied diffusion models and regression methods.

Specifically for FTTB/H diffusion, Briglauer and Gugler (2013) apply a logistic model to analyse actual data for household penetration in various countries. They present figures that confirm the sigmoid-shaped diffusion process. For some Asian countries with high deployment rates close to full national coverage, the authors can establish a saturation level of about $50 \%$. They suggest that this level holds for the other countries as well. They also find that the inflection point will be reached much later in the US and EU countries than in 
Asian countries that have already reached that point between 2007 and 2008. According to their estimation for the US as well as Northern and Eastern European countries, half of the saturation level will not be reached before 2015 and 2014 respectively, not to mention other European countries, which lag behind even further. Consistently, similar findings are drawn for the speed of diffusion. The growth rates for leading Northern European countries and the US are substantially lower than for Asian countries. The authors regard different levels of state aid, competition, and access regulation as the reasons for these differences in diffusion speed. They conclude that cost-based access regulation reduces broadband investments, albeit without statistically linking this finding to the diffusion analysis.

A recent study by Lin and Wu (2013) provides insights into the effects of multiple determinants for broadband diffusion. The idea of this study is to show that the significance of various factors differs for different stages of the diffusion process. The authors examine the stages of broadband development based on data gathered by the OECD for some 30 countries in the years 1997-2009. They use the Gompertz model because unlike the logistic model it does not require estimation of the total number of potential adopters for a certain time in a given country. For the panel regression, the authors apply generalized methods of moments (GMM) dynamic panel-data estimation developed by Arellano and Bond (1991), in order to account for the endogeneity problem caused by the lagged value of a dependent variable, namely the previous penetration. Based on Rogers (2003) they identify five categories of adopters and cluster them into three groups: innovators and early adopters, early majority, and late majority and laggards. In accordance with previous studies, the authors demonstrate that income, education, broadband price, platform competition, content, as well as previous broadband penetration, are significant determinants for broadband diffusion. However, they also find that the significance differs for the three categories of adopters, or stages of the diffusion process. The initial stage is determined by income, education and broadband price. This is consistent with the general observation that early adopters belong to wealthier and more educated groups of the population. More surprisingly, the broadband price has a positive significant coefficient during this stage, which suggests that broadband services are seen as a conspicuous good with a positive price elasticity of demand. Hence, in the early stage of broadband diffusion, higher prices do not seem to lower the demand. For the second stage, the authors identify both previous broadband penetration and platform competition to have the highest effect. The final stage of diffusion is characterised by a significant negative effect of broadband price while platform competition does not seem to matter. Therefore, affordable prices are much more relevant for the latecomers than competition between the various broadband technology platforms, namely DSL, cable, fibre, satellite and wireless. From their findings, the authors also deduce policy 
recommendations, which however appear to be too precipitant to be solid.

\subsection{The Effect of Government Intervention on NGA Investment and Penetration}

In addition to the studies on general determinants of broadband diffusion, a further body of literature benchmarks various regulatory instruments and other forms of government intervention with respect to their effects on NGA uptake (adoption) and investments.

\subsubsection{Effects on Adoption}

To predict the effects of regulation on broadband adoption, Crandall et al. (2013) perform a regression analysis on OECD data of broadband penetration levels in 28 countries for the years 2001 to 2010 . They build on the methods of an earlier study by Boyle, Howell and Zhang (2008), taking heteroskedasticity into account, but use more recent data and add further explanatory variables. The approach of the analysis is to test to what degree unbundling and line-sharing either shift the entire broadband diffusion curve or alter its slope. In confirmation of the results of previous studies, the authors arrive at a rather disappointing outcome for regulation. They find that there is evidence that unbundling regulation slows broadband penetration and reduces the slope of the diffusion curve. In addition, the authors predict that the effect of regulation is worse for fibre technology. They find it unlikely that fibre unbundling will provide net economic benefits. Due to the more complex issues of fibre unbundling, regulatory policies to grant network access to competitors most likely would raise deployment costs and increase the risk of regulatory error.

The effect of regulation on the adoption specifically of NGA services is the concern of an empirical assessment by Briglauer (2013). Based on panel data regarding connected NGA lines in all 27 EU member states for the years 2004 to 2012, his regression analysis shows that wholesale broadband access regulation has a substantial negative impact on NGA adoption. This outcome can be explained with reduced investment incentives for both regulated incumbent infrastructure operators and potential entrants. Existing wholesale regulation causes the expectation that cost-oriented access pricing of NGA networks will lower the profits and hence the net present value of infrastructure investments. Access regulation also typically ignores opportunity costs of real options and the asymmetric distribution of risks between investing operators and access-seeking entrants. Regulatory uncertainty impedes NGA investments even further. Briglauer also establishes that competition from mobile networks entails opposing effects. Fixed-line network operators might be incentivized to escape mobile competition by upgrading their networks to speeds 
which cannot be achieved with mobile broadband networks. On the other hand, mobile competition will eventually decrease the potential rents from NGA investments. For now, the escape competition effects dominate. Further, the analysis reveals a replacement effect with respect to 'old' broadband by which consumers are reluctant to switch to NGA unless its incremental benefits are large and transparent enough (consumer inertia). Finally, substantial network effects are found that result in an endogenous NGA adoption process that pushes the uptake of new ICT services. The larger the subscriber base becomes, the more related applications and content will be developed, which in turn leads to higher utility for consumers, and their willingness to adopt NGA services. Briglauer concludes that the Digital Agenda of the European Commission to reach $50 \%$ households with high-speed internet connections by 2020 seems in principle to be at odds with the EU sector-specific regulatory framework. However, the endogenous adoption process means that the target can still be reached if technologies other than FTTH/B are taken into account, no demand or supply side shocks occur, and no counter-effective policy incentives are implemented.

\subsubsection{Effects on Investments}

The finding that unbundling regulation has negative effects is in line with studies that estimate the relationship between regulation and investments.

In the context of net neutrality, Wallsten and Hausladen (2009) were among the first to discuss the effects of local loop unbundling on investment in new fibre networks in Europe. They regard net neutrality as another form of mandatory network sharing, and unbundling as the EU's general response to net neutrality. The interesting point of the study is the result that regardless of the effects of unbundling on net neutrality, regulation of networks can negatively impact investment incentives. The authors prove their point by demonstrating a simple correlation between the number of fibre broadband connections with the number of unbundled lines per capita in the form of either DSL over unbundled local loop or bitstream access. Controlling for GDP, they find that more unbundled lines are associated with fewer fibre broadband connections provided by both incumbents and entrants. On the other hand, there is a positive correlation between cable and fibre connections, suggesting that incumbents react to cable competition with investments in fibre broadband networks.

A more recent econometric study is provided by Grajek and Röller (2012), who base their analysis on a model that differentiates between investments of incumbents and entrants. The authors take endogeneity of regulation into account. This might arise when regulators base their decisions on the level of investments already made by network 
operators; regulation might not only have an effect on the investment decisions of operators, but also the investment decisions could affect regulation. The authors measure the level of infrastructure investments for 70 fixed-line telecom operators in $20 \mathrm{EU}$ member states during the years 1997 to 2006 . In order to determine the intensity of regulation, they make use of the detailed and comprehensive Plaut regulatory index, which seems to be more precise than relying on the number of wholesale products or the prices of local loop unbundling. As a result, the authors establish that access regulation discourages investment by incumbents and individual entrants alike. Entrants' total investment increases though, which suggests that easier access pushes entrants towards service based competition. Further, incumbents react to investments by entrants and invest more as entrants' total investment increases. The authors estimate for Europe a loss in industry investments of some EUR 16.4 billion over ten years due to access regulation. They also conclude that endogeneity of regulation in fact exists, resulting in a regulatory commitment problem. Finally they find that regulators respond differently to investments by incumbents as compared to those by entrants. While access regulation is not affected by investments of entrants, increased investments of incumbents result in expanded access regulation.

Another econometric model is used by Bacache et al. (2013) to investigate whether the ladder-of-investment hypothesis underlying the European regulatory approach holds true in reality. The ladder-of-investment theory claims that service-based competitors will climb up the ladder of regulatory wholesale products to eventually deploy their own networks and become infrastructure-based operators (Cave and Vogelsang, 2003; Cave, 2006). A typical scenario would be a competitor starting with wholesale products such as resale or bitstream access, then migrating towards local loop unbundling, and finally ending up with own access networks. Once entrants have gained a sufficiently large customer base, they can climb up the wholesale product ladder and gradually invest in their own infrastructure. The authors analyse wholesale data from 15 EU countries for the years 2002 to 2010 and reject the ladder-of-investment hypothesis. The empirical evidence does not show an impact of the number of unbundled lines on investment in new access infrastructure by new entrants. At the very most, there is a weak empirical support for a "short ladder" by means of which entrants move from bitstream access to local loop unbundling but do not invest further in own (fibre based) access infrastructure. Evidently, the study only proves that the regulatory reality in Europe is incompatible with the ladder-of-investment hypothesis. It could still be conceivable that a correct application of the ladder-of-investment would result in more investment by entrants if the regulatory agencies successively took rungs away, i.e. deregulated wholesale products over time, and hence set incentives for entrants to invest in infrastructure. 
Briglauer et al. (2013) find that the type of competition matters for the degree of investments in NGA networks. They quantify the effects of both service-based and infrastructure-based competition on the deployment of NGA in the $27 \mathrm{EU}$ member states during the period of 2005 to 2011 . Service-based competition is measured as the percentage of regulated and actually used wholesale broadband lines. According to the findings of the study, the more effective access regulation of DSL-based broadband is, the less investments are made in NGA. As a result, the ladder-of-investment hypothesis does not hold for NGA, and access regulation reduces investment incentives. Infrastructure-based competition has multiple opposing effects, resulting in a relationship between competition and investment that can be depicted with an inverted U-shaped curve (theorised by Aghion et. al, 2005). First, incentives to invest into NGA are low because these new infrastructures cannibalise profits from existing copper-based broadband networks. Second, with increasing competition incentives to invest increase as network operators now try to jump ahead of rivals. Third, as perfect competition approaches, potential rents are reduced and incentives to invest decline. In conclusion, the degree of inter-platform competition from mobile networks and cable operators also influences NGA deployment.

Comparing developed and developing countries, Gulati and Yates (2012) examine the effect of policy instruments and regulation on broadband diffusion. They find that there are differences in the impact of policy measures on broadband diffusion depending on the state of development of a country. Specifically, in developed countries, the degree of competition is not a statistically significant determinant for the uptake of broadband services, whereas it is in developing countries. The authors explain this result by the fact that the positive effect of competing over customers diminishes in the later stages of diffusion when the uptake rate begins to decrease. Also, the political structure (such as democracy) plays a more significant role in developing countries than in developed. The study finds a positive relationship between financial investment in ICT and broadband diffusion for both developing and developed countries. This is not surprising as it measures overall national expenditure in ICT, and it remains unclear to what extent the measured financial investment results from public financing and governmental incentives to invest.

The focus of a study by Montolio and Trillas (2013) lies on the question whether centralisation of public policies benefits broadband deployment. Public intervention in broadband internet access markets can be pursued by local authorities or on more centralised levels (such as federal state or EU level). While local governments might be better suited for the management of local rights of way, centralised institutions might be more efficient in dealing with cross-border issues. The study finds no positive relationship between 
broadband deployment and centralised public intervention (different forms of centralization are either irrelevant or have a negative impact).

\subsection{Summary of Empirical Findings}

Numerous empirical studies in the field of broadband diffusion unequivocally demonstrate several factors that are important to consider when judging on policies to enhance broadband availability. First, the diffusion of next generation broadband technology follows the sigmoid diffusion curve common for all forms of technological development. In the initial stage, uptake will be slow, and in the first years consumers will only show limited demand. It would be a mistake to see the slow uptake as a market failure that needed to be remedied with certain policy measures. In the long run, the maximum diffusion will be reached, no matter which strategies governments follow. For the penetration of NGA, it has been estimated that the saturation level is attained at about $50 \%$ of households. The only impact governmental policies generally will have is on the overall speed with which diffusion occurs, depicted by a more or less steep diffusion curve.

Second, there are many explanatory factors for the variation of diffusion speeds in different countries. Most of them relate to general socioeconomic circumstances and are outside of the scope of broadband policy. Especially the duration of the first stage of diffusion is influenced by income and GDP, but also education and population density play a major role. In the later stages, the level of the existing previous broadband penetration is important as well. There is a learning effect, and higher penetration in the early stages spills over to later stages and accelerates diffusion even more. Also, the availability of content, which makes a use case for consumers, is relevant. If the utility is clear for consumers, they are more likely to upgrade their network access. This has been described as consumer inertia and also been established as a factor for the New Zealand market in a household survey (Mirza and Beltrán, 2013).

Subsidies and other financial incentives can accelerate the deployment of new broadband access lines. It should be noted, however, that penetration will be stimulated more by demand-side than by supply-side incentives. Also, demand-side policies are mainly effective in the later stages of the diffusion. In light of these findings, the possible positive impact of financial aid to build up broadband network infrastructures seems to be questionable.

There has been a huge debate about the role of access regulation for the deployment 
of next generation access networks. Especially the European Union is led by the idea that competition drives technology and favours a strict regime of access regulation, although it has been pointed out that network operators might underinvest in the light of ex-ante regulation (Winkler and Baumgarten, 2009, p. 450). The empirics now clearly and unequivocally demonstrate that access regulation has in fact had a negative impact on the speed of broadband diffusion. Both the penetration speed and the efforts of infrastructure operators are being hampered by access regulation. The heavier access regulation is, the less progressively investments are made by incumbents and entrants alike. This clear empirical picture is also pertinent to NGA diffusion. If anything, access regulation slows down the deployment of NGA. In particular, free-riding problems stemming from an asymmetric distribution of risk, as well as regulatory uncertainty, diminish investment incentives. The ladder-of-investment hypothesis has been empirically disproven, although it could be argued that it has been applied wrongly by keeping all access regulation rungs (regulated wholesale products) in place.

More relevant seems to be the existence of inter-platform competition. Such competition to new fibre optical networks can stem from cable operators or high-speed data mobile networks. This form of competition is not induced by access regulation (such as local loop unbundling or bitstream access). Intra-platform competition, on the other hand, has been found to be less significant for network diffusion. Governments can nevertheless accelerate NGA uptake by providing a regulatory environment that favours inter-platform competition. For example, cost-effective licensing of LTE (4G) mobile spectrums could be an advantageous strategy. Effective competition from mobile technologies can induce broadband network operators to upgrade their networks in order to escape that competition.

\section{Situation of NGA development in New Zealand}

Internationally, New Zealand is not on the forefront of NGA uptake. Countries like Japan, South Korea, and Sweden have achieved impressive fibre access network uptakes in the last years, greatly pushed by massive state interventions and subsidies. But is New Zealand lagging behind? And what should or could be done about it?

The following sections briefly address some issues that are relevant for the development of next generation fibre access networks in New Zealand. Parallels to the development of broadband in the early 2000 s can be seen, with similar demand-side and supply-side factors playing a role. However, more research involving analysis of actual 
uptake figures would be needed to permit decisive conclusions.

\subsection{Parallels to the Development of Broadband}

The current situation in New Zealand is broadly comparable to the substitution from dial-up to broadband internet roughly ten years ago. At that time, public opinion expressed that New Zealand was lagging behind other countries in the uptake of broadband. However, the relatively slow uptake was due to specific factors of demand, and not to underinvestment in infrastructure.

As Howell and Obren (2003) point out, the substitution of dial-up internet access with DSL was (during the early 2000s) primarily observed in the business market. The residential broadband market showed a slow uptake although general internet connectivity of New Zealand's residents was large, with New Zealand consistently being among the top ten OECD countries. Consumers still seemed to have to learn about applications that made broadband connections necessary. The authors predicted that as more dial-up consumers learn about applications, and use them, the rate of information exchange will increase and broadband substitution will follow. Next to these learning effects, Howell (2003) found that dial-up products had a very high quality and were so cheap that consumers did not have incentives to switch to broadband yet. New Zealand exhibited no signs of market failure, and showed widespread coverage of broadband with DSL being available to $85 \%$ of the country's telecommunications customers. Also the prices for broadband were amongst the lowest within the OECD. However, the internet transfer volume was very low in relation to the high overall usage of the internet. This implied that there was a shortage of applications from which New Zealand's users could derive productivity or utility benefits. This demand-side problem could not have been addressed by infrastructure regulation. In other words, it was foreseeable that the emergence of bandwidth requiring applications would be necessary to drive increased broadband uptake.

The actual market development appears to confirm those predictions. New Zealand has achieved a level of broadband penetration which compares favourably to other OECD countries, and has recently even overtaken some of its peers. According to OECD statistics ${ }^{1}$ of June 2013, New Zealand has (with 29.5 fixed broadband subscriptions per 100 inhabitants) a penetration rate just slightly above OECD average (26.7\%), and is even followed by the USA (29.3\%), Japan (27.8\%), and Australia (25.6\%). As to fibre access networks, New Zealand's penetration level of $0.3 \%$ is far behind the leading countries like

1 OECD: http://www.oecd.org/sti/broadband/oecdbroadbandportal.htm. 
Korea $(23.3 \%)$, Japan $(19.1 \%)$, or Sweden $(11.6 \%)$, but comparable to many other economies, such as Germany (0.3\%), Austria (0.4\%), Australia $(0.5 \%)$, Italy $(0.5 \%)$, France $(0.6 \%)$, and Canada $(0.7 \%)$.

In sum, the question arises whether New Zealand is actually far behind within the OECD, and whether the seemingly slow uptake of high-speed broadband access is really a market failure that needs government intervention. As a government plan with subsidized private public partnerships to roll-out ultra-fast broadband (UFB) is already in progress, this question can only be discussed in terms of an ex-post assessment of the efficiency and effectiveness of this programme. Nevertheless, such an exercise would be a valuable contribution as it could highlight potential problems with UFB uptake in the future, and also might put expectations about the market for next-generation access into perspective. To evaluate the current situation, both demand-side and supply-side factors could be assessed, some of which are introduced in the following sections.

\subsection{Demand-side Factors of NGA uptake}

Consumers will switch to new internet access technology only if they expect to experience a more valuable use that at least outweighs the higher cost associated with the new technology (Howell, 2006). The higher bandwidth of ultra-fast broadband (UFB) internet connections are not as such an argument to switch, but rather applications which require higher speeds than presently available for a consumer. Speed of internet connections faces diminishing marginal returns. The advantage of an ultra-high speed internet connection is larger compared to a low speed internet connection than compared to a high speed connection. A consumer already having a broadband access of, say, up to $50 \mathrm{Mbit} / \mathrm{s}$ will less likely switch to UFB than a consumer who until previously could only get $1 \mathrm{Mbit} / \mathrm{s}$. As a result, one can expect a slower uptake rate of UFB than was seen in the case of the switch from dial-up to ADSL and cable broadband.

Hence, the main question from a demand-side perspective is which applications could drive demand for UFB, and make "normal" broadband redundant over time? The main application which currently requires most of the bandwidth is video streaming. Internet traffic analysed by a network solution company ${ }^{2}$ show that in the USA applications such as Netflix and Youtube are responsible for the major share in internet bandwidth usage, and have outrun peer-to-peer traffic in the last years. For the European and the Asia-Pacific regions, peer-to-peer still remains the strongest traffic generator. According to Sandvine, this suggests

2 Sandvine, 2013: https://www.sandvine.com/trends/global-internet-phenomena/ 
that users download many movies and TV series via peer-to-peer, but would be willing to change to paid services such as Netflix if they were available and comparably priced in the respective regions. Once similar services become more readily available in New Zealand, it can be expected that video streaming platforms will drive demand for higher broadband speeds in this country as well.

Video streaming can currently be seen as a selling argument for UFB, but presumably only for users who do not have access to medium-speed internet connections provided for by ADSL+ and VDSL (i.e. somewhere in the range of 16 to $50 \mathrm{Mbit} / \mathrm{s}$ ). This medium-speed range is sufficient for HD video streaming, and even several streams simultaneously. Netflix, for example, recommends an internet connection of $7 \mathrm{Mbit} / \mathrm{s}$ for Super HD and of $12 \mathrm{Mbit} / \mathrm{s}$ for $3 \mathrm{D} .{ }^{3}$ Although other factors than bandwidth may influence video quality, it is safe to say that medium-speed internet access is fairly sufficient even in a multiple person household. However, with the advancement of 4K TV screen technology, further bandwidth requirements can already be anticipated.

It seems to be very conceivable that the driving factor for UFB uptake in New Zealand is, under the current set of applications, the migration from low bandwidth broadband (ADSL) to higher bandwidths required for video streaming. Households that already have access to medium range broadband will be less likely switch to UFB as the marginal cost for doing so would not be countered by at least the same marginal benefit. This is because under the current situation, the marginal benefit would be close to zero for the average consumer. For existing medium-speed customers, the only viable argument to switch access products would be that UFB turns actually out to be cheaper than the conventional copper-based equivalents. An empirical analysis of recent UFB uptake data could show whether these assumptions are correct and apply to New Zealand. If mainly users that do not have access to medium-range broadband buy UFB internet access, while those that did have access are not inclined to pay for UFB, then this would indicate diminishing marginal returns of internet access speed, i.e. that users generally do not value more speed as much if the existing speed already satisfies their current needs.

A related question is how much the demand of new networks is dependent on the availability of applications. One could assume that there are two reciprocal network effects in place that determine the diffusion curve of ultra-fast broadband networks: the availability of bandwidth-hungry applications drives the demand for high-speed broadband access; but also the uptake of high-speed broadband access enables the market for applications that rely on

3 Netflix: help.netflix.com/en/node/306 
such uptake. It has been observed for Japan, for example, that although a nationwide highspeed fibre access network exists with very wide coverage and uptake, the cloud-based applications that require the available bandwidth (speed) are not yet in sight (Kushida, 2013). The reason is that application providers, such as Youtube, Netflix, and Dropbox, focus on global markets and the broadband access speed that can be expected to exist in the major parts of the world. This market mechanism should be even more significant for the much smaller economy of New Zealand. Further, applications that depend on latency rather than volume will not benefit from an increase in speed of the access network alone if it can be assumed that remote regions like New Zealand remain disadvantaged due to the distance data packages need to travel (Obren and Howell, 2010).

The demand-side factors of UFB uptake are difficult to address by government intervention. Legal obstacles to providing bandwidth demanding applications do not appear to exist in New Zealand. The relevant market players (such as broadcasting companies, film producers, and video streaming portals) just have to come up with offers that would utilize increasing bandwidth availability in the country. However, as said above, applications which require the incremental bandwidth of UFB compared to medium scale broadband are still to be developed. This problem is not peculiar to New Zealand, and only time will tell which bandwidth-hungry applications will be demanded.

Demand-side interventions by governments, such as investments in education for computer and internet literacy, certainly have the potential to enhance demand for broadband. However, such financial support will not be able to shift demand from medium range broadband to UFB. This would require stimulation of the supply of bandwidth demanding applications. Subsidies of media companies would be an option; however, as there are no apparent signs of market failure in this respect, any government intervention should be considered with caution as it could potentially distort markets more than it would vitalize the economy. Specific research of internet application producing industries would be required to make conclusive recommendations.

\subsection{Supply-side Factors of NGA deployment}

Globally, governments have focused on supply-side factors in their aim to enhance availability of ultra-fast broadband (UFB). There are two main approaches that also have significance in New Zealand: subsidies of UFB networks and sector-specific regulation of telecommunications. 


\subsubsection{Subsidies of UFB networks}

In 2009, the New Zealand government started the UFB initiative with the aim to roll out ultra-fast broadband to $75 \%$ of the population within ten years. To lower the risk for private investment, public private partnerships (PPP) were set up, which are funded with a NZD 1.5bn government investment. The PPPs are managed by the government owned Crown Fibre Holdings (CFH). CFH agreed with four operators to roll out fibre access networks in 33 priority areas that mainly comprise densely populated regions. Chorus, the structurally separated network arm of the former incumbent Telecom, is responsible for roughly $70 \%$ of the roll-out areas, while the three remaining operators deploy fibre in the regions of Whangarei (Northpower), Christchurch (Enable), and Waikato (Ultrafast Fibre). CFH maintains individual agreements about the deployment details and timelines with the respective network operators. Further, $\mathrm{CFH}$ acquired shares in Chorus and in the aforementioned local fibre subsidiaries of the other partners, referred to as Local Fibre Companies (LFC). By virtue of the PPP agreements, the fibre access network operators do not sell fibre connections to consumers directly, but rather provide wholesale products for about 50 New Zealand internet retail service providers. The LFCs are rewarded on the basis of providing broadband access on the wholesale level in a particular area (premises passed).

By July 2013, the LFCs had deployed fibre access to 229,633 premises (which equals about $20 \%$ of the initiative's objective; CFH, Annual Report 2013, p. 15). On the demandside, just under 10,000 customers had signed-up for connections (including private households, businesses, and schools). This penetration level of about $4 \%$ of connectable premises is in line with CFH's expectations.

However, some issues with the government plan seem to be unresolved. Chorus currently needs NZD 3200 on average to pass each premise, and it appears not to be able to cover its operating costs with the current level of CFH funding, which gives rise to doubts whether the UFB initiative provides sufficient means to reach its targets (Sadowski et al., 2013 , p. 76). The main issue will be whether the residential uptake will follow the deployment rate, and whether the network operators will be able to sell sufficient UFB wholesale products to cover their cost. An important aspect is that the main roll-out regions are overlapping to a large extent with areas where medium-speed broadband networks are already available, including the recently built up VDSL network of Chorus (FTTN). An analysis of recent demand-side data could clarify the substitution effects between existing broadband infrastructure and newly deployed fibre networks, and possibly shed light on the financing 
issues of the four LFCs in New Zealand.

\subsubsection{Regulatory Environment}

While New Zealand's UFB networks are not subject to sector-specific access regulation, the network operators act in an environment that is determined by pricing rules for copper-based wholesale products. The Commerce Commission issues standard term determinations (STDs) that set out the terms on which telecommunications service providers (namely Chorus) must offer wholesale services regulated under the Telecommunications Act 2001 to other telecommunications service providers. The regulated fixed-line wholesale products include copper-based local loop unbundling (ULL), line-sharing access to the lowfrequency band of the copper local loop, and bitstream access. Currently, only Chorus is regulated, being the structurally separated network arm of the former monopolist Telecom.

The UFB networks are, as such, unregulated. Access to wholesale products for retailers are part of the agreements with $\mathrm{CFH}$. By virtue of these arrangements, UFB network operators cannot offer retail services themselves. As a result, structural separation between UFB networks and retail services exists throughout New Zealand.

The regulatory environment likely has an effect on the deployment of UFB networks. It is clear that New Zealand's approach favours UFB over copper-based high-speed internet access. As a result, the regulatory environment might cause inconsistencies (Heatley and Howell, 2010; Howell, 2012). Currently, the wholesale prices for access to copper are higher compared to equivalent fibre wholesale prices. Therefore, retail internet service providers are able to offer fibre-based broadband connections that should be more attractive for consumers than the DSL-based counterparts (Sadowski et al., 2003, p. 75). Although this situation perfectly fits the objective of a fast UFB uptake, it might cause adverse effects for competing broadband networks that are not subsidized, such as the recently rolled out VDSL network of Chorus, and the $4 G$ mobile networks that make use of auctioned frequencies.

Moreover, the incentives of retail service providers to offer UFB based internet products are not clear cut. Because of vertical separation they are not invested in network deployment and are able to choose between different wholesale platforms to offer fixed broadband connections; that is DSL, cable, and UFB. Also, network operators face the question as to whether competition with existing broadband lines is a sensible strategy in the first phase of deployment, or should be delayed to the later stages. Especially, Chorus potentially could cannibalize its existing VDSL lines by deploying parallel fibre access lines. 
On the other hand, the cost of deploying UFB should be lowest in those regions which already have high-speed internet access. This creates incentives to invest in those areas first. It will be interesting to see how Chorus deals with this dilemma. A recent amendment to the agreement between $\mathrm{CFH}$ and Chorus might give an indication: amongst others, Chorus will have greater flexibility in the deployment of UFB in areas where Chorus already has an extensive fibre footprint. ${ }^{4}$

In sum, the regulatory environment is complex, but also an essential factor of the deployment of UFB in New Zealand. An appraisal of its effects would best be achieved by looking at actual developments. An analysis of data on network distribution and retail prices could shed light on the impact of regulation on UFB deployment in New Zealand.

\section{Conclusion}

The review of empirical research on broadband diffusion revealed several factors that are relevant for the deployment of next generation access (NGA) networks. First, the diffusion of next generation broadband technology follows the sigmoid diffusion curve common to all forms of technological development. In the initial stage, uptake will be slow, and in the first years of deployment of a new technology, such as ultra-fast broadband, consumers will only show limited demand. It would be a mistake to see the slow uptake as a market failure, which needed to be remedied with certain policy measures. In the long time perspective, the maximum diffusion will be reached, no matter which strategies governments follow. The only impact politics generally will have is on the overall speed with which diffusion occurs, depicted by a more or less steep diffusion curve. Second, there are many explanatory factors for the variation of diffusion speeds in different countries. Most of them relate to general socioeconomic circumstances and are outside of the scope of broadband policy. Especially the duration of the first stage of diffusion is influenced by income and GDP, but also education and population density play a major role. The availability of content which makes a use case for consumers is relevant as well. If the utility is clear for consumers, they are more likely to upgrade their network access.

Government intervention in form of subsidies and other financial incentives can accelerate the deployment of new broadband access lines, but penetration will be stimulated more by demand-side than by supply-side incentives. In light of these findings, the possible positive impact of financial aid to build up broadband network infrastructures seems to be

4 CFH, 2014: http://www.crownfibre.govt.nz/2014/03/chorus-crown-fibre-holdings-agree-package-ufbimprovements/ 
questionable. As to regulation, empirics now clearly and unequivocally demonstrate that access regulation has in fact had a negative impact on the speed of broadband diffusion. Both the penetration speed and the efforts of infrastructure operators are being hampered by access regulation. This clear empirical picture also fits to NGA diffusion. If at all, access regulation slows down the deployment of NGA. Much more relevant seems to be the existence of inter-platform competition. Such competition to new fibre optical networks can stem from cable operators or high-speed data mobile networks. For example, cost-effective licensing of LTE (4G) mobile spectrum could be an advantageous strategy. Effective competition from mobile technologies can induce broadband network operators to upgrade their networks in order to escape that competition.

These elements are applicable to the situation in New Zealand and its ultra-fast broadband network that is currently being built. It can be questioned whether New Zealand is actually far behind within the OECD, and whether the seemingly slow uptake of high-speed broadband access was really a market failure that needed government intervention. To evaluate the current situation, both demand-side and supply-side factors should be assessed. From a consumer perspective, video streaming can currently be seen as the main selling argument for ultra-fast broadband (UFB), but presumably only for users who do not have access to medium-speed internet connections provided for by copper-based internet access. Although other factors than bandwidth may influence video quality, it is safe to say that medium-speed internet access is currently sufficient even in a multiple person household.

What presumably matters most for consumer demand is not the absolute speed of internet connections, but rather the increase in speed in relation to the existing access technology. The higher the existing broadband speed, the less likely consumers will be to switch to new technology, absent applications requiring that increase in speed. Therefore, it seems conceivable that the driving factor for UFB uptake in New Zealand is, under the current set of applications, the migration from low bandwidth broadband (ADSL) to higher bandwidths required for video streaming. An empirical analysis of recent UFB uptake data could show whether these assumptions apply to New Zealand.

The regulatory environment likely has an effect on the deployment of UFB networks. It is clear that New Zealand's approach favours UFB over copper-based high-speed internet access. Currently, the wholesale prices for access to copper are higher compared to equivalent fibre wholesale prices. As a result, retail internet service providers are able to offer fibre-based broadband connections that should be more attractive for consumers than the 
DSL-based counterparts. Although this situation perfectly fits the objective of a fast UFB uptake, it might cause adverse effects for competing broadband networks that are not subsidized, such as the recently rolled out VDSL network of Chorus, and the $4 \mathrm{G}$ mobile networks that make use of auctioned frequencies.

Moreover, the incentives of retail service providers to offer UFB based internet products are not clear cut. Because of vertical separation they are not invested in network deployment and are able to choose between different wholesale platforms to offer fixed broadband connections. The regulatory environment is complex, but also an essential factor of the deployment of UFB in New Zealand. An appraisal of its effects would best be achieved by looking at actual developments. An analysis of data on network distribution and retail prices could shed light on the impact of regulation on UFB deployment in New Zealand. 


\section{References}

Aghion, P., Bloom, N., Blundell, R., Griffith, R., \& Howitt, P. (2005). Competition and innovation: an inverted-U relationship. Quart. J. Econ. 120, 701-728.

Arellano, M., \& Bond, S. (1991). Some tests of specification for panel data: Monte Carlo evidence and an application to employment equations. The Review of Economic Studies, 58(2), 277-297.

Bacache, M., Bourreau, M., \& Gaudin, G. (2013). Dynamic Entry and Investment in New Infrastructures: Empirical Evidence from the Fixed Broadband Industry. Review of Industrial Organization, 1-31. doi:10.1007/s11151-013-9398-4

Bass, F. M. (1969). A new product growth for model consumer durables. Management Science, 15(2), 215-227.

Belloc, F., Nicita, A., \& Alessandra Rossi, M. (2012). Whither policy design for broadband penetration? Evidence from 30 OECD countries. Telecommunications Policy, 36(5), 382-398. doi:10.1016/j.telpol.2011.11.023

Bouckaert, J., van Dijk, T., \& Verboven, F. (2010). Access Regulation, Competition, and Broadband Penetration: An International Study. Telecommun. Policy, 34(11), 661671. doi:10.1016/j.telpol.2010.09.001

Boyle, G., Howell, B., \& Zhang, W. (2008). Catching Up in Broadband Regressions: Does Local Loop Unbundling Really Lead to Material Increases in OECD Broadband Uptake? ISCR Working Paper. Retrieved from http://ssrn.com/abstract=1184339

Briglauer, W. (2013). The Impact of Regulation and Competition on the Adoption of FibreBased Broadband Services: Recent Evidence from the European Union Member States. Retrieved from http://epub.wu.ac.at/3942/

Briglauer, W., Ecker, G., \& Gugler, K. (2013). The impact of infrastructure and service-based competition on the deployment of next generation access networks: Recent evidence from the European member states. Information Economics and Policy, 25(3), 142-153. doi:10.1016/j.infoecopol.2012.11.003

Briglauer, W., \& Gugler, K. (2013). The deployment and penetration of high-speed fiber networks and services: Why are EU member states lagging behind? Telecommunications Policy, 37(10), 819-835. doi:10.1016/j.telpol.2013.05.003

Cava-Ferreruela, I., \& Alabau-Muñoz, A. (2006). Broadband policy assessment: A crossnational empirical analysis. Telecommunications Policy, 30(8-9), 445-463. 
Cave, M. (2006). Encouraging Infrastructure Investment via the Ladder of Investment. Telecommunications Policy, 30, 223-237.

Cave, M., \& Vogelsang, I. (2003). How Acces Pricing and Entry Interact. Telecommunications Policy, 27, 717-727.

CFH - Crown Fibre Holdings (2013). Annual Report. Retrieved from http://www.crownfibre.govt.nz/wp-content/uploads/2011/10/CFH-Annual-Report2013.pdf

Crandall, R. W., Eisenach, J. A., \& Ingraham, A. T. (2013). The long-run effects of copperloop unbundling and the implications for fiber. Telecommunications Policy, 37(4-5), 262-281. doi:10.1016/j.telpol.2012.08.002

Dauvin, M., \& Grzybowski, L. (2013). Estimating broadband diffusion in the EU using NUTS 1 regional data. Telecommunications Policy. doi:10.1016/j.telpol.2013.05.006

Dekimpe, M. G., Parker, P. M., \& Sarvary, M. (1998). Staged Estimation of International Diffusion Models. Technological Forecasting and Social Change, 57, 105-132.

Denni, M., \& Gruber, H. (2007). The Diffusion of Broadband Technology: the Role of Competition. Evidence from the US. Communications \& Strategies, 68, 139-158.

Distaso, W., Lupi, P., \& Manenti, F. M. (2005). Platform Competition and Broadband Uptake: Theory and Empirical Evidence from the European Union (Industrial Organization). EconWPA. Retrieved from http://econpapers.repec.org/scripts/a/abstract.pf? $\mathrm{f}=/$ paper/wpawuwpio/0504019.htm

Fildes, R., \& Kumar, V. (2002). Telecommunications demand forecasting--a review. International Journal of Forecasting, 18(4), 489-522.

Golder, P. N., \& Tellis, G. J. (2004). Going, Going, Gone: Cascades, Diffusion and Turning Points of the Product Life Cycle. Marketing Science, 23(2), 207-218.

Grajek, M., \& Röller, L.-H. (2012). Regulation and Investment in Network Industries:

Evidence from European Telecoms. Journal of Law and Economics, 55(1), 189-216.

Gregg, J. V., Hassel, C. H., \& Richardson, J. T. (1964). Mathematical trend curves: An aid to forecasting. Edingburgh: Oliver \& Boyd.

Grosso, M. (2006). Determinants of broadband penetration in OECD nations. In Australian Communications Policy and Research Forum. Retrieved from https://networkinsight.org/verve/_resources/GrossoM.pdf

Gruber, H., \& Verboven, F. (2001). The Diffusion of Mobile Telecommunications Services in 
The European Union. European Economic Review, 45(3), 577-588.

Gulati, G. J., \& Yates, D. J. (2012). Different paths to universal access: The impact of policy and regulation on broadband diffusion in the developed and developing worlds. Telecommunications Policy, 36, 749-761.

Heatley, D. \& Howell, B. (2010). Structural Separation and Prospects for Welfare-Enhancing Price Discrimination in a New 'Natural Monopoly' Network: Comparing Fibre Broadband Proposals in Austrlia and New Zealand. ISCR Working Paper. Retrieved from http://www.iscr.org.nz

Howell, B. (2003). Building Best Practice Broadband in New Zealand: Bringing Infrastructure Supply and Demand Together. ISCR Working Paper BH03 06. Retrieved from http://www.iscr.org.nz

Howell. B. (2006). Competition, Regulation and Broadband Diffusion: The Case fo New Zealand. ISCR Draft Paper. Retrieved from http://www.iscr.org.nz

Howell. B. (2012). Competition and Regulation Policy in Antipodean Government-Funded UltraFast Fibre Broadband Markets. Telecommunications Policy Research Conference Paper. Retrieved from http://ssrn.com/abstract=2031292

Howell, B., \& Obren, M. (2003). Telecommunications Usage in New Zealand: 1993-2003. ISCR Working Paper BH03 02. Retrieved from http://www.iscr.org.nz

Karaçuka, M., \& Çatik, A. N. (2012). Diffusion of Telecommunication Services in Turkey. Ege Academic Review, 12(4), 497-510.

Kushida, K. E. (2013). Public Private Interplay for Next Generation Access Networks: Lessons and Warnings from Japan's Broadband Success. Communications \& Strategies, 91(3), 13-34.

Lee, M., \& Cho, Y. (2007). The Diffusion of Mobile Telecommunications Services in Korea. Applied Economics Letters, 14, 477-481.

Lee, S., Marcu, M., \& Lee, S. (2011). An empirical analysis of fixed and mobile broadband diffusion. Information Economics and Policy, 23(3-4), 227-233. doi:10.1016/j.infoecopol.2011.05.001

Lin, M.-S., \& Wu, F.-S. (2013). Identifying the determinants of broadband adoption by diffusion stage in OECD countries. Telecommunications Policy, 37(4-5), 241-251. doi:10.1016/j.telpol.2012.06.003

Meade, N., \& Islam, T. (2001). Forecasting The Diffusion of Innovations: Implications for Time Series Extrapolation. In J. S. Armstrong (Ed.), Principles of forecasting. Boston: Kluwer Academic Publishers. 
Meade, N., \& Islam, T. (2006). Modelling, and Forecasting The Diffusion of Innovation - A 25Year Review. International Journal of Forecasting, 22(3), 519-545.

Michalakelis, C., Dede, G., Varoutas, D., \& Sphicopoulos, T. (2010). Estimating diffusion and price elasticity with application to telecommunications. Netnomics, 11, 221-242.

Mirza, F., \& Beltrán, F. (2013). Drivers and Barriers to the Uptake of a FTTH Ultra-Fast Broadband in New Zealand. TPRC 41: The 41st Research Conference on Communication, Information and Internet Policy. Retrieved from http://ssrn.com/abstract=2241514

Montolio, D., \& Trillas, F. (2013). Regulatory federalism and industrial policy in broadband telecommunications. Information Economics and Policy, 25(1), 18-31. doi:10.1016/j.infoecopol.2013.01.002

Obren, M., \& Howell, B. (2010). The Tyranny of Distance Prevails: HTTP protocol latency and returns to fast fibre internet access network deployment in remote economies. ISCR Working Paper. Retrieved from http://www.iscr.org.nz/f609,17429/The_Tyrant_Lives_v3_Nov21.pdf

Robertson, A., Soopramanien, D., \& Fildes, R. (2007). Segmental new-product diffusion of residential broadband services. Telecommun. Policy, 31(5), 265-275. doi:10.1016/j.telpol.2007.03.006

Rogers, E. M. (2003). Diffusion of innovations. New York: Free Press.

Rouvinen, P. (2006). Diffusion of Digital Mobile Telephony: Are Developing Countries Different? Telecommunications Policy. 30.

Sadowski, B., Howell, B., \& Nucciarelli, A. (2013). Structural Separation and the Role of Public-Private Partnerships in New Zealand's UFB Initiative. Communications \& Strategies, 91, 57-80.

Sundqvist, S., Frank, L., \& Puumalainen, K. (2005). The Effects of Country Characteristics, Culture Similarity and Adoption Timing on the Diffusion of Wireless Communications. Journal of Business Research, 58, 107-110.

Tsuji, M., Shinohara, S., \& Akematsu, Y. (2012). Empirical Analysis of Factors Promoting Broadband Deployment in OECD 30 Countries (SSRN Scholarly Paper No. ID 2032305). Rochester, NY: Social Science Research Network. Retrieved from http://papers.ssrn.com/abstract=2032305

Vanston, L. (2002). Forecasts for internet/online access. In D. G. Loomis \& L. D. Taylor (Eds.), Forecasting the Internet: Understanding the Explosive Growth of Data Communications (pp. 45-58). Dordrecht: Kluwer Academic Publishers. 
Wallsten, S. (2006). Broadband and Unbundling Regulations in OECD Countries (Working paper No. 314). Retrieved from http://econpapers.repec.org/scripts/a/abstract.pf? $\mathrm{f}=/$ paper/regwpaper/314.htm;terms=wallsten+2006+broadband

Wallsten, S., \& Hausladen, S. (2009). Net Neutrality, Unbundling, and their Effects on international Investment in Next-Generation Networks. Review of Network Economics, 8(1), 90-112.

Winkler, K. E., \& Baumgarten, G. (2009). The Framework for Network Access and Interconnection. In C. Koenig et al. (Eds.), EC Competition and Telecommunications Law, 2nd ed. (pp. 421-469). Alphen aan den Rijn, The Netherlands: Kluwer Law International.

Wu, F., \& Chu, W. (2010). Diffusion Models of Mobile Telephony. Journal of Business Research, 63, 497-501. 